\title{
Single-Session Mechanical Thrombectomy of Lower Extremity Deep Venous Thrombosis Using the ClotTriever System: A Single-Institution Experience
}

\author{
Vibhor Wadhwa ${ }^{1}$ Anuj Malhotra ${ }^{1}$ Andrew Kesselman ${ }^{1}$ \\ ${ }^{1}$ Division of Interventional Radiology, New York-Presbyterian/Weill \\ Cornell Medical Center, New York, New York United States \\ Address for correspondence Vibhor Wadhwa, MD, Division of \\ Interventional Radiology, New York-Presbyterian/Weill Cornell \\ Medical Center, 525 East 68th St, New York, NY 10065, USA \\ Arab J Intervent Radiol 2021;5:71-75. \\ (e-mail: viw9032@nyp.org).
}

\begin{abstract}
Purpose The aim of this study was to provide a single-center experience in utilization of the ClotTriever mechanical thrombectomy system in acute and subacute deep vein thrombosis (DVT).

Methods Retrospective review was performed for all patients who underwent DVT thrombectomy using the ClotTriever system at a single academic medical center. Data recorded included age, gender, etiology, laterality of DVT, fluoroscopy time, perioperative parameters, type of anesthesia, concomitant usage of thrombolytics and/or stents. Follow-up Doppler ultrasounds or diagnostic venograms were reviewed, and time of reintervention (if performed) and Villalta scores were recorded.

Results Ten patients were included in the study, with $6 / 10$ female (60\%), mean age 54.6 years (range: $24-80$ ). Eight patients presented with acute iliofemoral DVT, one patient with subacute iliofemoral DVT, and one patient with subacute inferior vena cava thrombosis. Only one patient required concomitant intraprocedural thrombolytic administration, and four patients underwent same-session venous stent placement. Technical success was achieved in $90 \%$ patients and no procedure-related adverse events were observed. Reintervention within 30 days was required in two (20\%) patients at 12 and 13 days. No patient required intensive care unit (ICU) stay, and mean

\section{Keywords}

- deep vein thrombosis

- mechanical thrombectomy

- ClotTriever

- postthrombotic syndrome postprocedure hospital length of stay was 2.3 days. On follow-up (mean: 214 days), five patients had no evidence of DVT and four patients showed chronic postthrombotic changes in femoropopliteal veins not requiring intervention.

Conclusion The ClotTriever mechanical venous thrombectomy system demonstrates high efficacy in removal of thrombus from lower extremities in a single session, often obviating the need for thrombolytic therapy with low reintervention rates and short postprocedure hospital stay.
\end{abstract}

published online February 2, 2022
DOI https://doi.org/ 10.1055/s-0041-1742088. ISSN 2542-7075.
(C) 2022. The Pan Arab Interventional Radiology Society. All rights reserved.

This is an open access article published by Thieme under the terms of the Creative Commons Attribution-NonDerivative-NonCommercial-License, permitting copying and reproduction so long as the original work is given appropriate credit. Contents may not be used for commercial purposes, or adapted, remixed, transformed or built upon. (https://creativecommons.org/ licenses/by-nc-nd/4.0/)

Thieme Medical and Scientific Publishers Pvt. Ltd., A-12, 2nd Floor, Sector 2, Noida-201301 UP, India 


\section{Introduction}

Lower extremity deep vein thrombosis (DVT) can be a cause of significant morbidity, with acute symptoms often progressing to postthrombotic syndrome (PTS), which is sequala of chronic venous insufficiency developing after DVT. To prevent clot propagation in acute DVT, the first line of therapy remains therapeutic anticoagulation. ${ }^{1}$ Anticoagulation alone, however, does not lead to active thrombus removal, and long-standing thrombus within the veins can lead to venous hypertension, valve incompetence, and inflammatory reaction to the thrombus. This is thought to be the mechanism for the development of PTS, and therefore, thrombus removal is advocated in selected patients to provide symptomatic relief and reduce the incidence of PTS. ${ }^{2} \mathrm{~A}$ multitude of endovascular thrombectomy devices are available that vary in rates of technical success and may be limited by need for thrombolytics. The ClotTriever system is a largebore suction thrombectomy platform designed for thrombus removal without the need for concomitant thrombolytics. Although there is robust data on catheter-directed thrombolysis in acute DVT management, there is relative paucity of safety and efficacy data on purely mechanical thrombectomy without the use of lytics. The purpose of this study is to provide a preliminary single-center experience in utilization of the ClotTriever mechanical thrombectomy (Inari Medical Inc, Irvine, California, United States) system in acute $(<14$ days) and subacute (14-28 days) DVT.

\section{Materials and Methods}

Institutional board review approval was obtained for this retrospective study.

\section{Patient Population}

Retrospective chart review was performed for all patients who underwent DVT thrombectomy using the ClotTriever system from 9/2018 to 7/2020 (from device availability to time of study) at a single academic medical center. Data recorded included age, gender, etiology, laterality of DVT, acuity of DVT (acute $<14$ days, subacute 14-28 days, chronic $>28$ days of clinical symptoms), fluoroscopy time, perioperative parameters, type of anesthesia, concomitant usage of thrombolytics and/or stents. Technical success was defined as adequate thrombus evacuation leading to restoration of antegrade flow determined on postprocedural venography without the use of adjunct thrombectomy devices. Follow-up time period was recorded, Doppler ultrasounds/venograms (if reintervention was required) were reviewed, and Villalta scores recorded. Postthrombotic changes were defined as sonographic signs of decreased venous diameter, intraluminal material, wall thickening, or synechiae/webbing/bands.

\section{Technical Details}

The ClotTriever system includes two components: a proprietary $13 \mathrm{~F}$ sheath and the thrombectomy device. The system is advanced over a 0.035 -inch wire. The sheath features a

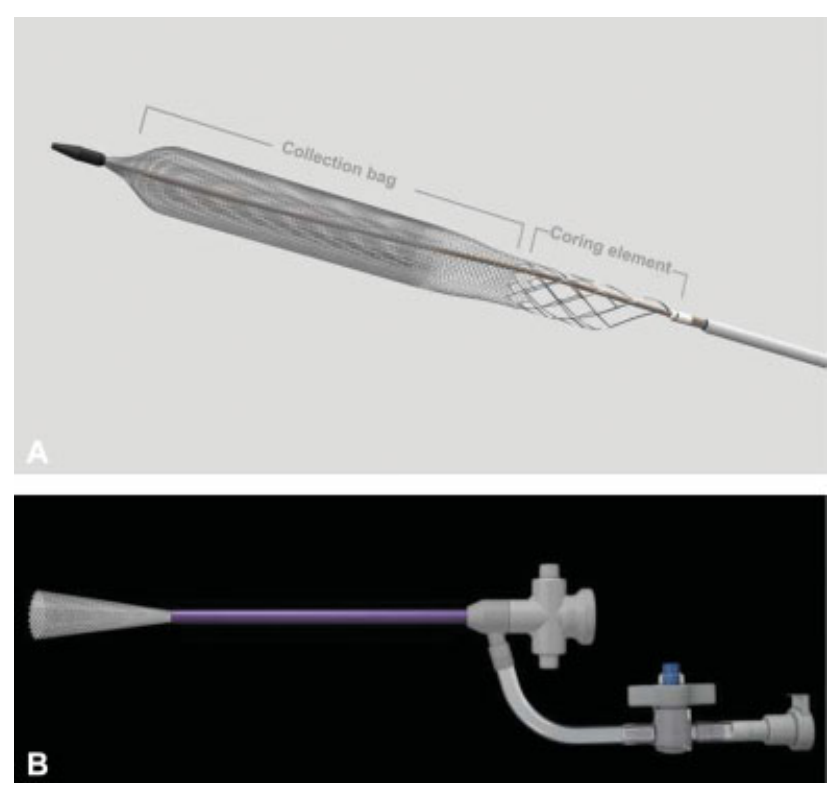

Fig. 1 The ClotTriever system comprising the thrombectomy device (A) and proprietary $13 \mathrm{~F}$ sheath (B).

self-expanding nitinol funnel at its tip. The catheter comprises a nitinol coring element at its leading edge, and a braided thrombus collection bag at its trailing edge ( - Fig. 1).

All procedures were performed in the interventional radiology suite with moderate sedation or general anesthesia by board certified interventional radiologists with average 3.8 years of postfellowship experience in interventional management of venous disease. Patients were anticoagulated prior to and during the procedure, and heparin administration was guided by intraoperative measurement of activated clotting time (target $>250$ ). The vein was accessed using a standard micropuncture set under ultrasound guidance. Initial venogram was performed using $5 \mathrm{~F}$ sheath and catheter to determine the clot burden. A 0.035 inch wire was advanced through the occluded venous segment into the internal jugular vein or subclavian vein. The $5 \mathrm{~F}$ sheath was then upsized and exchanged for $13 \mathrm{~F}$ ClotTriever sheath with deployment of the funnel. The catheter was introduced through the sheath and advanced superiorly into the inferior vena cava (IVC), with the nitinol coring element cranial to the occluded venous segment. The coring element and collection bag was deployed and the system retracted caudally under fluoroscopic guidance until the coring element reached the funnel tip of the sheath. The collection bag was then closed and catheter removed. All clot was then removed from the collection bag prior to reinsertion for a second pass. Repeat venogram was performed via the sheath to document resolution of clot burden, and additional interventions (venoplasty or stenting) were performed per operator preference (-Figs. 2 and $\mathbf{3}$ ).

\section{Data Analysis}

All data was stored and analyzed in Excel 2016 (Microsoft Corp, Redmond, Washington, United States). Two sample $t$ test was used to compare groups and $p$-value of $<0.05$ was considered significant. 

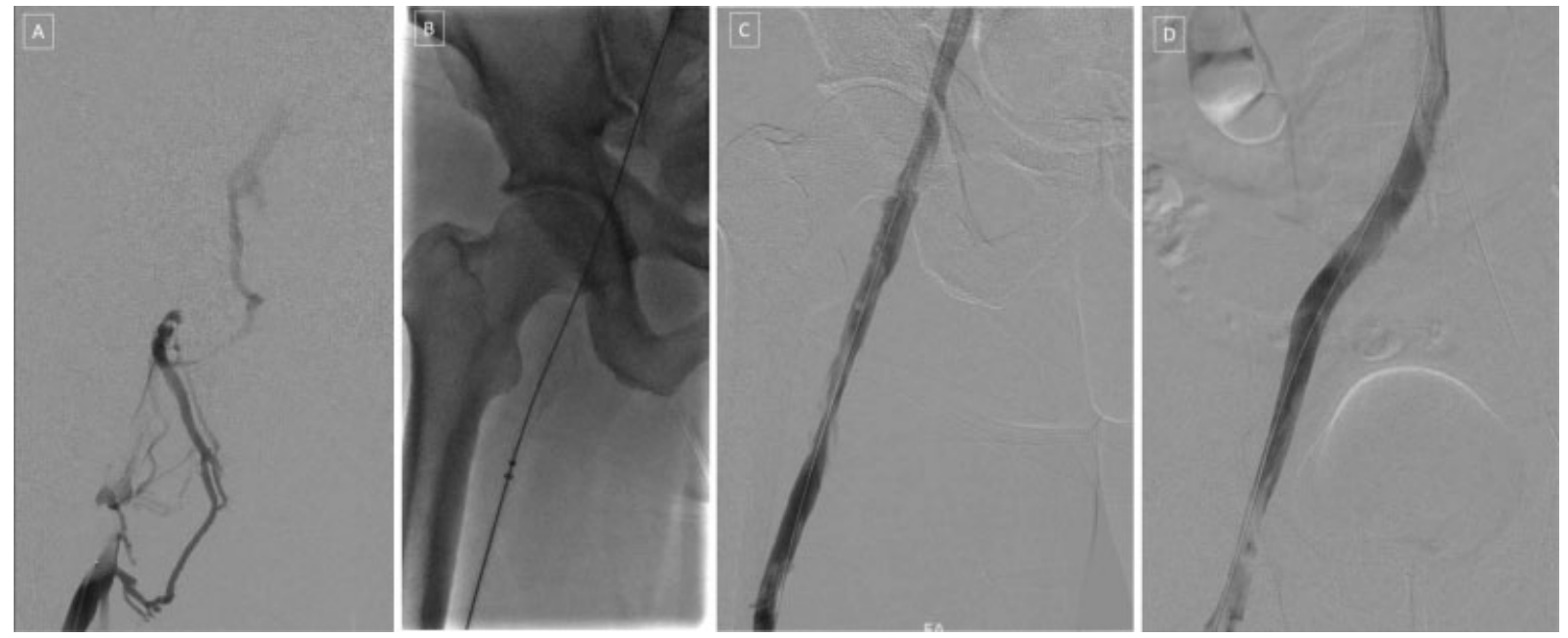

Fig. 2 A 40-year-old patient with acute left lower extremity deep venous thrombosis. Fluoroscopic and digital subtraction venographic images obtained with patient in prone position and access via the left popliteal vein. Initial DSV (A) showing acute thrombus within the iliofemoral segment, with opacification of collaterals. Subsequent fluoroscopic image (B) showing the ClotTriever device deployed over the wire within the iliofemoral segment. DSV after three passes of ClotTriever (C) showing restoration of in-line flow. (D) Stent placed in the iliac venous segment at the site of May-Thurner lesion. DSV, digital subtraction venogram.
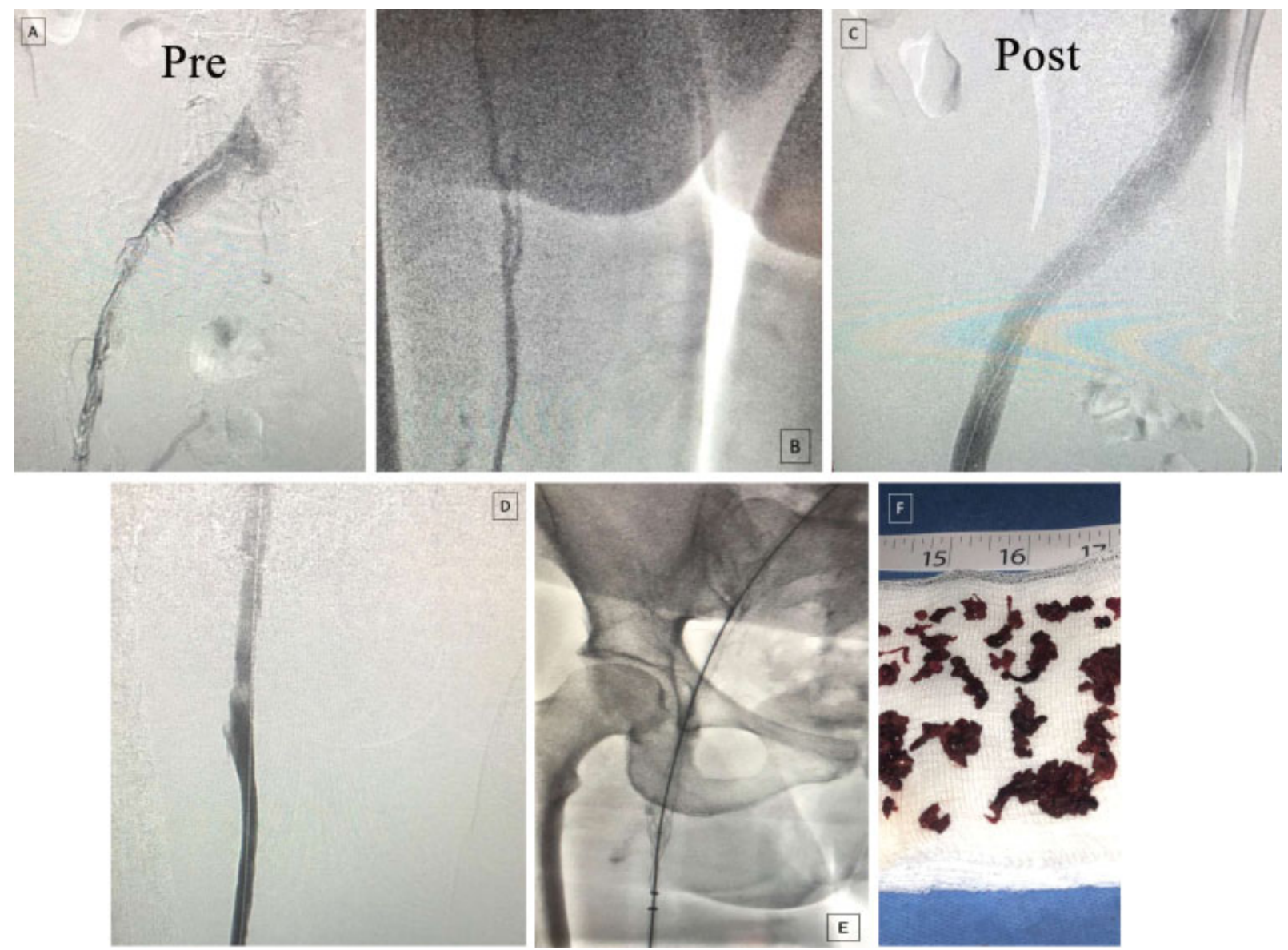

Fig. 3 A 80-year-old patient with myelofibrosis presenting with acute left lower extremity deep vein thrombosis. Fluoroscopic and digital subtraction venographic images before $(A, B)$ and after (C, D) mechanical thrombectomy and stent placement. Fluoroscopic image (E) showing the ClotTriever device deployed over the wire within the iliofemoral vein. Acute thrombus (F) extracted from the thrombotic veins. 


\section{Results}

Ten patients were included in the study (-Table 1), with 6/10 female (60\%), mean age 54.6 years (range: 24-80). The mean ( \pm standard deviation) fluoroscopy time was 34.01 ( \pm 15) minutes and dose-area product of $67.48( \pm 34.9) \mathrm{Gy}^{*} \mathrm{~cm}^{2}$. Eight patients presented with acute iliofemoral DVT, one patient with subacute iliofemoral DVT, and one patient with subacute IVC thrombosis. The popliteal and femoral veins were accessed in 9/10 and 1/10 patients, respectively. Only one patient required concomitant intraprocedural thrombolytic administration, and four patients underwent same-session venous stent placement. Restoration of antegrade flow was achieved in all patients and technical success was achieved in $90 \%$ patients without any procedure-related adverse events. There was no significant difference in pre- and postoperative hemoglobin (10.38 vs. 10.08 , $p=0.78)$ and creatinine ( 0.87 vs. $0.86, p=0.95)$. Reintervention within 30 days was required in two (20\%) patients at 12 and 13 days, both using the AngioJet rheolytic pharmacomechanical thrombectomy system. ${ }^{3}$ No patient required intensive care unit (ICU) stay and mean postprocedure hospital length of stay was 2.3 days. Follow-up imaging was available for 9 patients, with a mean follow-up interval (defined as date of procedure to date of most recent ultrasound/venogram) of 230 days (range: 48-569). Five (of 9) patients had no evidence of recurrent DVT. Four (of 9) patients had chronic postthrombotic changes in femoropopliteal veins that did not require further intervention and only one patient had clinical signs and symptoms of mild PTS(Villalta score 11). Mean follow-up Villalta score of patients was 3.

\section{Discussion}

The ClotTriever is an over-the-wire mechanical thrombectomy system designed to core and extract thrombus off the venous wall. It has the ability to capture and remove large clot burden from large caliber vessels in a single session. The device obviates the need for thrombolytics, thereby eliminating ICU stay, which is a major advantage of this device. Although thrombolytics are effective in removing thrombus, they are associated with higher incidence of bleeding. Although the incidence of thrombolyticrelated bleeding in endovascular procedures was as low as $1.7 \%$ in the ATTRACT trial, ${ }^{4}$ the rate of adverse bleeding due to lytics is commonly reported to be between 3 and $5 \%$.

The present study showed high technical success and safety in all patients (-Table $\mathbf{1}$ ). Only one patient required concomitant use of lytics, which was intraprocedural on operator preference. Two patients with May-Thurner anatomy and IVC atresia, respectively, required reintervention due to persistent clinical symptoms, and both were accomplished using AngioJet. The technical results from present study are similar to those reported by Benarroch-Gampel et al, who also reported high technical success and low reintervention rates with the ClotTriever device. ${ }^{5}$ In addition to mitigating thrombolytic use, the device also has potential financial benefits. It avoids the cost of thrombolytic drugs (tissue plasminogen activator), ICU stay, and potential repeat intervention after removal of thrombus. These benefits do come at the expense of increased fluoroscopy time and dose. The mean fluoroscopy time and dose-area product in the present study were 34.01 minutes and of $67.48 \mathrm{~Gy}^{*} \mathrm{~cm}^{2}$, respectively, which are higher than those reported by Lim et al for lower extremity DVT thrombolysis, the median fluoroscopy time and dose-area product in this study being 16.35 minutes and $9.2 \mathrm{Gycm}^{2}$, respectively. ${ }^{6}$ However, it must be noted that in the present series, four patients underwent concomitant stenting; the mean fluoroscopy time for stented patients was 41.15 and for nonstented cases was 29.36 minutes.

Table 1 Characteristics of patients who underwent mechanical thrombectomy using ClotTriever system

\begin{tabular}{|c|c|c|c|c|c|c|c|c|}
\hline $\begin{array}{l}\text { Age/ } \\
\text { gender }\end{array}$ & $\begin{array}{l}\text { Contributing } \\
\text { etiology }\end{array}$ & Segments & Lytics & Stent & $\begin{array}{l}\text { Technical } \\
\text { success }\end{array}$ & $\begin{array}{l}\text { Postop } \\
\text { LOS }\end{array}$ & $\begin{array}{l}\text { Most } \\
\text { recent } \\
\text { follow-up }\end{array}$ & Reintervention \\
\hline $41 / M$ & May-Thurner & Left iliofemoral & No & Yes (CIV) & Yes & 6 & 386 & No \\
\hline $44 / F$ & Liposarcoma & IVC & No & No & Yes & 2 & NA & \\
\hline $74 / F$ & Idiopathic & Left iliofemoral & No & No & Yes & 1 & 569 & No \\
\hline $50 / F$ & May-Thurner & Left iliofemoral & No & No & Yes & 2 & 450 & $\begin{array}{l}\text { Yes, } 427 \text { days, } \\
\text { venoplasty }\end{array}$ \\
\hline $64 / M$ & May-Thurner & Left iliofemoral & No & $\begin{array}{l}\text { Yes } \\
\text { (CIV, CFV) }\end{array}$ & Yes & 2 & 91 & Yes, 12 days, Angiojet \\
\hline $49 / F$ & TKR & Left iliofemoral & No & No & Yes & 1 & 246 & No \\
\hline $24 / M$ & IVC atresia & Left iliofemoral & Yes & No & No & 1 & 48 & Yes, 13 days, Angiojet \\
\hline $77 / F$ & Myelofibrosis & Left iliofemoral & No & $\begin{array}{l}\text { Yes } \\
(\mathrm{CIV}, \mathrm{CFV})\end{array}$ & Yes & 4 & 84 & No \\
\hline $43 / \mathrm{M}$ & COVID-19 & Right iliofemoral & No & No & Yes & 1 & 99 & No \\
\hline $80 / F$ & $\begin{array}{l}\text { Myelofibrosis, } \\
\text { May-Thurner }\end{array}$ & Left iliofemoral & No & $\begin{array}{l}\text { Yes } \\
\text { (CIV, CFV) }\end{array}$ & Yes & 3 & 98 & No \\
\hline
\end{tabular}

Abbreviations: CFV, common femoral vein; CIV, common iliac vein; COVID-19, coronavirus disease 2019; IVC, inferior vena cava; LOS, length of stay; TKR, total knee replacement. 
There are several limitations to this study. First, the retrospective nature of the study and lack of control group prevent comparisons and control for extraneous factors affecting outcomes. Second, patient selection for the ClotTriever system is not uniform among the practitioners and is largely based upon operator's choice. Third, there are limitations of the device itself, that is, large access size (13F) and relatively contraindicated via the internal jugular vein approach (to avoid nitinol coring element and collection bag passing through the heart). Finally, the small sample size is a limiting factor in generalizing these results, while being cognizant that this is a real-world nonindustry-sponsored study.

In conclusion, in this early real-world experience, the ClotTriever mechanical venous thrombectomy system appears to have a high efficacy in the removal of thrombus from lower extremities in a single-session setting, and may obviate the need for thrombolytic therapy with low reintervention rates and short postprocedure hospital stay. Future large-scale studies should be performed to further establish the role of this system in patients with DVT and candidates for thrombectomy.

Ethical Approval

Approved by Institutional Review Board.

Funding

None.

\section{Conflicts of Interest}

None declared.

\section{References}

1 Kearon C, Akl EA, Ornelas J, et al. Antithrombotic therapy for VTE disease: CHEST guideline and expert panel report. Chest 2016; 149(02):315-352

2 Haig Y, Enden T, Grøtta O, et al; CaVenT Study Group. Postthrombotic syndrome after catheter-directed thrombolysis for deep vein thrombosis (CaVenT): 5-year follow-up results of an open-label, randomised controlled trial. Lancet Haematol 2016;3 (02):e64-e71

3 Angiojet $^{\mathrm{TM}}$ Peripheral Thrombectomy System [Internet]. www. bostonscientific.com [cited 2020 Oct 17]. Accessed December 20, 2021 from: https://www.bostonscientific.com/en-US/products/ thrombectomy-systems/angiojet-thrombectomy-system.html

4 Vedantham S, Goldhaber SZ, Julian JA, et al; ATTRACT Trial Investigators. Pharmacomechanical catheter-directed thrombolysis for deep-vein thrombosis. N Engl J Med 2017;377(23): 2240-2252

5 Benarroch-Gampel J, Pujari A, Aizpuru M, Rajani RR, Jordan WD, Crawford R. Technical success and short-term outcomes after treatment of lower extremity deep vein thrombosis with the ClotTriever system: a preliminary experience. J Vasc Surg Venous Lymphat Disord 2020;8 (02):174-181

6 Lim CS, Waseem S, El-Sayed T, et al. Patient radiation exposure for endovascular deep venous interventions. J Vasc Surg Venous Lymphat Disord 2020;8(02):259-267 